\title{
S'est-il vraiment passé quelque chose d'important en Afrique du Sud en février 2018 ?
}

Alain Dubresson and Philippe Gervais-Lambony

\section{(2) OpenEdition}

\section{Journals}

Electronic version

URL: http://journals.openedition.org/echogeo/15286

DOI: 10.4000/echogeo.15286

ISSN: 1963-1197

\section{Publisher}

Pôle de recherche pour l'organisation et la diffusion de l'information géographique (CNRS UMR 8586)

\section{Electronic reference}

Alain Dubresson and Philippe Gervais-Lambony, « S'est-il vraiment passé quelque chose d'important en Afrique du Sud en février 2018 ? », EchoGéo [Online], Sur le Vif, Online since 13 March 2018, connection on 19 April 2019. URL : http://journals.openedition.org/echogeo/15286 ; DOI : 10.4000/ echogeo. 15286

This text was automatically generated on 19 April 2019

\section{cc) (†) $\odot$}

EchoGéo est mis à disposition selon les termes de la licence Creative Commons Attribution - Pas d'Utilisation Commerciale - Pas de Modification 4.0 International 


\title{
S'est-il vraiment passé quelque chose d'important en Afrique du Sud en février 2018 ?
}

\author{
Alain Dubresson and Philippe Gervais-Lambony
}

1 Le 14 février 2018, Jacob Zuma, président de la République d'Afrique du Sud depuis 2009, a présenté sa démission. Le lendemain, Cyril Ramaphosa a été élu par l'Assemblée Nationale pour le remplacer jusqu'à la fin prévue du mandat présidentiel au printemps 2019 et il est évident qu'il sera alors candidat à la présidence de la République. L'opinion sud-africaine suit depuis longtemps le feuilleton des errements politiques de Jacob Zuma, l'opinion internationale a observé les derniers soubresauts avec attention, une presse souvent très élogieuse sur la victoire et le personnage de Cyril Ramaphosa a vu là un virage important : l'arrivée au pouvoir d'un politicien de haute stature et rassurant pour remplacer un chef de l'État impliqué dans tant de scandales politico-financiers.

On pense souvent que ce qui est nouveau est meilleur que ce qui s'achève, c'est humain... Dans le cas présent, est-il permis d'en douter ? On aura tout entendu sur la corruption du régime et du clan Zuma, sa famille, ses proches, très liée au pouvoir exercé sur l'appareil d'État d'une famille d'affairistes d'origine indienne, les Gupta (Bhorat et. al., 2017), sur l'état économique d'un pays dont la croissance économique a drastiquement ralenti depuis 2009 et qui subit les ravages du chômage (au moins $30 \%$ de la population active) et de la pauvreté (avec environ $55 \%$ des Sud-africains vivant sous le seuil national de pauvreté) (Gervais-Lambony, 2017).

Dans ce contexte, un changement à la tête de l'État sud-africain est certes un événement national important et il est normal de le saluer, normal aussi d'y voir une bonne nouvelle. Mais faut-il y voir un virage majeur? Après tout, c'est bien le vice-président du même Jacob Zuma qui devient président de la République (conformément à la Constitution). Cyril Ramaphosa appartient bien au même parti politique, au pouvoir depuis 1994, l' African National Congress (ANC); il en est même, depuis décembre 2017, le nouveau président. Ce sont aussi deux hommes qui appartiennent pratiquement à la même génération qui se succèdent au pouvoir: même si le nouveau président a une petite 
dizaine d'années de moins que son prédécesseur, tous deux sont d'anciens proches de Nelson Mandela. Certes leurs parcours sont différents: Jacob Zuma, qui a connu des années de prison, a été un membre puissant de l'ANC en exil, alors que Cyril Ramaphosa a été un leader syndicaliste luttant de l'intérieur du pays, avant de devenir un homme politique. Tous deux tiraient ou tirent leur légitimité de leur passé de lutte contre le régime de l'apartheid : ce sont des hommes du post-apartheid. La question est de savoir si cela ne les met pas en décalage avec une société sud-africaine qui est peut-être déjà audelà : post-post-apartheid (un chiffre justifie à lui seul cette question : $47 \%$ des Sudafricains ont moins de 25 ans et sont donc nés après la fin de l'apartheid).

Il n'y a, en tous cas à la tête de l'État, ni changement de génération, ni changement de parti politique. C'est l'ANC qui a exigé et obtenu la démission de Jacob Zuma. Avec quelle principale motivation? Changer de politique sociale et économique, servir mieux le pays et lutter contre la corruption ou simplement se débarrasser d'un personnage devenu bien encombrant et dont le maintien en place risquait de faire perdre au parti les élections de 2019 ?

On peut douter de la réalité d'un virage politique majeur. Le dessin publié par le célèbre cartooniste Zapiro (ci-dessous) après la démission de Jacob Zuma (symbolisé par la pomme de douche, référence à ses propos malheureux tenus lors de son procès pour viol en 2006) résume au fond un sentiment qu'il est possible de partager : si nous sommes à la fin d'une parenthèse malheureuse et aux effets dévastateurs, alors on peut penser plus à un pas en arrière qu'en avant...

Illustration 1 - Dessin publié suite à la démission de Jacob Zuma

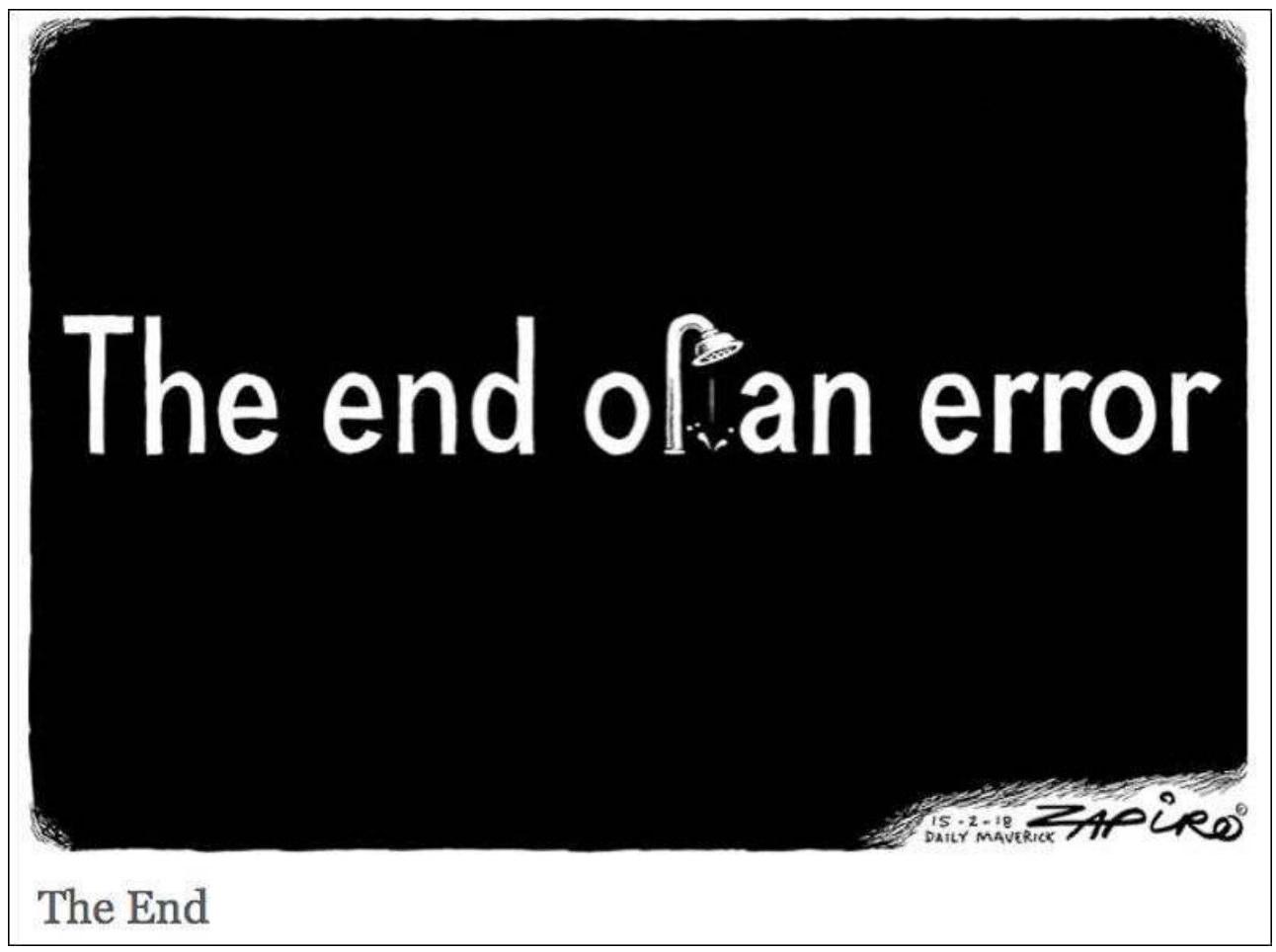

Auteur : Zapiro, 15/02/18.

Quels changements majeurs d'orientation politique sont annoncés par le nouveau président? Le principal est la lutte contre la corruption. Très symboliquement, au 
moment où sont rédigées ces lignes, sont actuellement recherchés par la police sudafricaine l'un des frères Gupta et l'un des fils Zuma. Mais on ne peut pas faire comme si la corruption en Afrique du Sud était apparue avec l'arrivée au pouvoir de Jacob Zuma, ni comme si elle allait disparaître du jour au lendemain après son départ. De quelle corruption parle-t-on d'ailleurs? Celle d'une partie de la classe politique au pouvoir est réelle et profonde, et la défiance des électeurs sud-africains face à l'ANC y est liée. Mais la corruption est aussi vécue au quotidien, c'est l'ensemble de l'administration de l'État, à toutes les échelles, qui est concernée par le problème. Depuis le policier qui reçoit quelques billets pour ne pas sanctionner un automobiliste par une amende ou qui rackette le travailleur étranger sans papiers jusqu'au fonctionnaire municipal qui accepte de l'argent pour faire approuver un projet immobilier...

\section{Corruption, capitalisme et État en Afrique du Sud : des relations... ambiguës}

7 Au sommet de l'État sud-africain, la relation entre milieux d'affaires et pouvoir politique est ancienne. Elle est antérieure à l'Afrique du Sud telle qu'on la connaît aujourd'hui : Cecil Rhodes, gouverneur de la province du Cap, était un milliardaire en même temps qu'un homme politique, et il mettait la politique au service direct de ses intérêts économiques. Le régime d'apartheid était aussi au service du développement d'une élite économique afrikaner, un de ses objectifs affichés.

8 Et depuis la fin de l'apartheid, jamais, ni sous la présidence Mandela (qui dès 1994 a fait du développement des investissements étrangers une priorité), ni sous la présidence Mbeki les liens n'ont été coupés entre pouvoir politique et milieux d'affaires. On s'est beaucoup interrogé sur la nature de ces liens, sur l'existence ou non d'une alliance ou d'une coalition de croissance entre les élites politiques de l'ANC et les détenteurs blancs du capital privé et sur la responsabilité de ces relations dans la situation actuelle. Les uns privilégient la notion de pacte des élites (Bond, 2000; Webster and Adler, 1999), accord conclu entre les grandes firmes blanches encore concentrées en conglomérats multisectoriels à la fin de la décennie 1980 et les éléments les plus nationalistes de l'ANC. Ce compromis aurait abouti, d'une part, à la préservation des intérêts du capitalisme blanc, contrebalancée par un accès d'élites noires au capital des entreprises accompagné d'une libéralisation en faveur du marché, d'autre part, à l'acceptation des réformes politiques par le patronat blanc en échange du maintien de la rentabilité des entreprises. D'autres mettent en exergue le poids structurel du complexe minéralo-énergétique (Fine and Rustomjee, 1996), ancré dans un partenariat historique entre pouvoir d'État et capitalisme privé, dont les intérêts seraient convergents, voire confondus, ce que l'accès au pouvoir des dirigeants de l'ANC n'aurait pas modifié.

9 Une autre interprétation des relations entre ANC et élite économique est possible (Seekings and Natrass, 2011) : depuis 1994, le pouvoir d'État sud-africain aurait surtout cherché à discipliner le capitalisme blanc (lequel est d'ailleurs hétérogène) et à le transformer, dans un climat de méfiance généralisée. Depuis l'arrivée de l'ANC au pouvoir, des relations spécifiques entre pouvoir d'État et entreprises auraient ainsi été créées, constituant une forme curieuse d'embedded autonomy (autonomie enchâssée). L'État serait soucieux et respectueux de la rentabilité des firmes qui redistribuent des revenus à des individus «non-blancs » et participent au changement social, mais il ne 
s'impliquerait plus lui-même dans la production, contrairement aux anciens gouvernements d'apartheid. Il serait plus tolérant envers toutes les formes de chevauchement politique et tous les mécanismes de conversion de capital constitutifs du néopatrimonialisme (Lodge, 2014). La mise en œuvre du Black Economic Empowerment marquerait ainsi le début d'une intrication entre élites politiques noires et entreprises dont on comprend bien qu'elle peut être interprétée en termes de corruption ou tout du moins que la limite entre l'une et l'autre est fragile. Quoi qu'il en soit et quelle qu'en soit l'origine, le Black Economic Empowerment avait pour objectif de faire émerger un nouveau capitalisme noir et des élites économiques susceptibles d'innover, à moyen terme, en matière de choix de société. Or Cyril Ramaphosa lui-même est une des plus belles réussites de cette politique (Butler, 2013).

Encadré 1 - Les multiples visages du nouveau président

Cyril Ramaphosa, né en 1952 à Soweto, militant anti-apartheid pendant ses études de droit et à deux reprises emprisonné dans les années 1970, a été fondateur en 1982 puis secrétaire général du National Union of Mineworkers, puis fondateur du COSATU (la confédération des syndicats sudafricains qui fut un acteur majeur de la lutte contre l'apartheid) en 1985. Élu secrétaire général de l'ANC en 1992, il a été le principal négociateur du compromis politique qui a conduit au changement de régime politique en Afrique du Sud. Il a ensuite été président de l'Assemblée en 1994, c'est donc sous sa houlette qu'a été rédigée la Constitution de 1996. Pressenti pour succéder à Nelson Mandela mais évincé par Thabo Mbeki, il démissionne de ses fonctions politiques en 1997 pour se lancer dans les affaires et créer sa compagnie, la New Africa Investments Limited devenue depuis le Shanduka Group. La fortune de Cyril Ramaphosa est estimée à 800 millions de dollars US, ce qui ferait de lui un des plus riches sud-africains. Le Shanduka Group a des participations dans les secteurs énergétique, minier, immobilier, financier et dans les télécommunications, il contrôle aussi la branche sud-africaine de la chaîne McDonald's. L'homme d'affaires a cependant continué à siéger au Comité Exécutif National de l'ANC où il a été réélu en 1997 et jusqu'à 2012. En décembre 2012, avec le soutien de Jacob Zuma, il est élu vice-président de l'ANC. C'est aussi Jacob Zuma qui l'appelle à la vice-présidence du pays, sans doute en partie pour rassurer les milieux économiques et la vieille garde du parti, en partie aussi parce qu'il vaut mieux avoir ses ennemis près de soi...

10 Un regard en arrière sur les relations entre capitalisme et État conduit donc à se demander si la corruption (avérée) d'un clan Zuma-Gupta est vraiment l'enjeu central du changement en cours en Afrique du Sud. Le rand se renforce, la bourse (Johannesburg Stock Exchange) va mieux, les milieux d'affaires se réjouissent, les investisseurs étrangers vont peut-être revenir, le pays va peut-être "émerger » à nouveau ou enfin... Mais on a connu tout cela déjà, et a-t-on vraiment abouti à une société plus « juste »? Qu'est-ce qui va changer pour les plus fragiles, les plus exclus du système? Inégalités sociales et spatiales, jeunesse sans emploi, territoires abandonnés, secteur para-public en crise, immigrés martyrisés, violences multiformes... va-t-on voir disparaitre ces fléaux?

\section{Émerger ou ne pas émerger ?... est-ce que telle est la question?}

11 Il faut remonter un peu le fil du temps pour mieux comprendre à quel point les fractures politiques et sociales sont profondes. Elles traversent la société sud-africaine comme le 
parti au pouvoir et elles sont très liées à des valses - hésitations sur les politiques économiques et la définition même de l'ANC et de son rôle à la tête de l'État, au-delà des questions de personnes.

Jusqu'en 2008, tout semblait aller dans le sens d'une émergence de l'économie sudafricaine: taux de croissance annuel robuste d'environ $5 \%$, monnaie stable, balance commerciale bénéficiaire, faible endettement, début de diversification industrielle. Aujourd'hui le rand est affaibli, la balance commerciale déficitaire, l'endettement fort. Depuis 2009, la croissance n'a jamais retrouvé son niveau antérieur ; le taux de croissance n'a été que de $0,3 \%$ en 2016, début 2017 l'économie sud-africaine serait entrée en récession mais finalement le résultat 2017 est estimé positif $(+1,3 \%)$. Cette situation correspond à une phase de profonde transformation économique : l'industrie contribuait pour $20 \%$ de l'économie nationale en 1997 contre moins de $15 \%$ en 2016 ; les services, devenus dominants, fournissent plus de $50 \%$ des emplois mais ne peuvent absorber la main-d'œuvre sous-qualifiée. Cette transformation résulte du modèle de transition et de la mise en œuvre d'un nouveau mode de régulation hybride qui mêle néo-libéralisme et redistribution pour répondre au double impératif d'impulsion d'une croissance forte par l'ouverture accélérée au marché mondial et de réallocation de ressources au bénéfice de ceux qui furent victimes de l'ancien régime raciste. La combinaison entre croissance et redistribution a cependant été fluctuante dans le temps, balançant entre les deux pôles selon un mouvement oscillant, ce qui rend complexe toute analyse de la trajectoire économique sud-africaine, ou en tout cas nécessite de la considérer avec un certain recul temporel.

13 Après une brève phase redistributive de type keynésien (Reconstruction and Development Programme, RDP), le gouvernement de l'ANC s'engage en 1997 dans un auto-ajustement structurel néo-libéral (Growth, Employment and Redistribution, GEAR), privilégiant l'insertion compétitive dans le marché mondial accompagnée d'une restructuration générale des entreprises et du secteur public. Le retour à une redistribution un peu plus équitable est prôné d'abord en 2006, avec l'Accelerated and Shared Growth Initiative for South Africa (ASGISA), puis en 2010 avec le New Growth Path.

Ces fluctuations traduisent les vives tensions internes à l'ANC, les luttes entre des courants très divisés, par exemple sur la définition de l'État développemental. La notion de developmental state est mobilisée par tous les acteurs publics, mais il n'y a aucun accord sur son contenu ainsi qu'en témoignent les atermoiements sur le sort des entreprises publiques, trois des «quatre grandes », Transnet, Eskom et Denal ayant été restructurées alors que Telkom a été en partie privatisée. Ces oscillations / changements expriment également des modifications de rapports de force au sein de la coalition de réforme sudafricaine, les grandes firmes privées et l'ANC ayant finalement marginalisé les syndicats et le parti communiste. Un fait majeur demeure: l'internationalisation rapide de l'économie sud-africaine. Celle-ci résulte d'un double mouvement de flux de capitaux, vers l'extérieur pour les grandes firmes nationales devenues de plus en plus globales, vers le territoire national pour les firmes étrangères occupant à la fois des niches délaissées par la recomposition des anciens conglomérats, comme Anglo American ou Rembrandt, et des créneaux nouveaux, surtout dans les services. Cette internationalisation a des conséquences, d'une part, sur la structure productive sud-africaine, avec l'émergence d'un complexe minéralo-énergétique "plus» (Southall, 2010) à forte composante de services, d'autre part, sur les discours et les pratiques souvent contradictoires concernant le secteur public et para public. Aujourd'hui, ce dernier rassemble 717 entreprises 
représentant $30 \%$ de l'investissement total, mais les plus grandes entreprises, fers de lance de l'État développemental censés assurer à l'État le contrôle des secteurs clés de l'économie et être des outils de transformation sociale, sont devenues un boulet financier pour le gouvernement (Tsheola et al., 2013). En 2017, leur dette extérieure pèse $39 \%$ de celle de l'État, les garanties de ce dernier équivalent à $11 \%$ du PIB et $50 \%$ des recettes annuelles. Le débat sur leur avenir est ainsi relancé, y compris au sein de l'ANC, et ce n'est pas par hasard si Cyril Ramaphosa, revêtant ses habits neufs de président, a inscrit l'action sur les entreprises publiques dans les 12 priorités de son discours à la Nation du 16 février 2018. Au-delà de ces priorités immédiates, c'est en réalité tout le modèle de transition mis en place depuis 1994 qui est à repenser, d'autant plus que depuis la fin des années 2000 , on assiste à une reprimarisation économique sous l'effet de la demande mondiale, et en particulier asiatique, en minerais et métaux de base dont l'Afrique du Sud est abondamment pourvue.

Encadré 2 - Eskom, un exemple emblématique de la crise des entreprises publiques

Premier producteur africain d'électricité, Eskom Holdings SOC Ltd est une entreprise publique en monopole, verticalement intégrée et détenue à 100 \% par l'État sud-africain. Ébranlé en 2008 par des ruptures locales de la fourniture d'électricité, puis contraint à pratiquer des coupures tournantes jusqu'en 2015, ce champion national a frôlé le désastre technique et, début 2018, est proche du naufrage financier. Il a fallu une première intervention de l'État, en 2014, pour recapitaliser l'entreprise, puis une seconde en janvier 2018 pour lui permettre d'assurer ses dépenses courantes durant deux mois. Les surcoûts financiers du programme de renforcement de la puissance installée, qui s'achève avec 5 ans de retard, et le montant considérable de la dette ont conduit les agences internationales de notation à dégrader les notes d'Eskom. Or, l'entreprise bénéfice d'une garantie de l'État et sa détresse financière pèse désormais sur l'ensemble de la dette publique et sur la crédibilité du Trésor sud-africain, les agences de notation ayant par ailleurs dégradé la note souveraine de l'Afrique du Sud fin 2017.

Pour comprendre pourquoi ce fleuron du capitalisme d'État sud-africain est en crise, il faut entrer dans la boîte noire des rapports entre la construction du pouvoir d'État, avant, pendant et après l'apartheid, et celle d'Eskom, née sous forme d'une simple commission en 1923, corporatisée en 1987 et convertie en entreprise publique en 2001. Les dysfonctionnements techniques du réseau électrique et les difficultés financières résultent d'abord des errements de la politique énergétique du gouvernement de l'ANC dans les années 2000, qui ont privé Eskom de stratégie industrielle et marqué une décennie perdue en matière de réformes du secteur électrique. Plus fondamentalement, ils révèlent les limites et impasses $d u$ régime technopolitique néopatrimonial d'Eskom, devenue un puissant outil du Black Economic Empowerment et de la redistribution de la rente charbonnière ( $85 \%$ de la production électrique proviennent de centrales à charbon) à des élites affairistes de l'ANC. Or, Eskom est au centre de gravité du triangle progressivement construit depuis 1923 et sans cesse renforcé, qui interconnecte d'une part le gouvernement de l'Afrique du Sud, les firmes privées et les entreprises publiques, et, d'autre part, l'ensemble de ces trois éléments au complexe minéraloénergétique. En affectant les trois sommets du triangle et le fonctionnement de tout le complexe minéralo-énergétique, la crise d'Eskom provoque des réactions instables en chaîne dont les effets de retour sont également négatifs pour l'entreprise : sa fragilisation ébranle tout l'édifice politique (Jaglin et Dubresson, 2016). 


\section{Des inégalités toujours plus fortes et un changement désormais possible du paysage politique sud-africain}

15 Si l'Afrique du Sud a peut-être été un pays émergent, elle ne l'est sans doute plus. Et les fractures de la société sud-africaine restent immenses même si, depuis 1994, 8 millions de personnes ont gagné un accès à l'eau courante, près de 3 millions de logements ont été construits et plus de 4,3 millions de foyers ont été connectés au réseau de distribution d'électricité. En contrepoint, l'Afrique du Sud est l'un des pays les plus inégalitaires du monde : le coefficient de Gini, 0,64, est l'un des plus élevés, les $10 \%$ les plus riches captent $65 \%$ du revenu national contre $0,6 \%$ pour les $10 \%$ les plus pauvres, $1 \%$ des Sud-africains concentrent $25 \%$ de ce même revenu et selon les dernières enquêtes, les inégalités sont plus fortes aujourd'hui qu'en 1994. La pauvreté monétaire augmente depuis 2011, 62 \% des Noirs étant classés comme pauvres en 2015.

Certes, un changement social majeur a bel et bien eu lieu : l'émergence d'une nouvelle élite et surtout d'une "classe moyenne" noire qui représente aujourd'hui environ 12 millions d'individus, soit 1 Sud-africain sur 5 . Il y a donc eu des gagnants de la période post-apartheid. Mais des perdants aussi, dans les quartiers les plus pauvres des villes et dans les anciens bantoustans. La situation de ces derniers est une des conséquences de l'absence de véritable réforme foncière : à peine $10 \%$ des terres qui auraient dû l'être ont été redistribuées ; dans les anciens bantoustans, 12 millions de Sud-africains sont toujours à l'écart de l'économie nationale. Pauvreté et chômage, concentrés dans les espaces perdants, sont des facteurs explicatifs forts de la crise du contrat social post-apartheid. Des signes en démontrent la gravité : explosions récurrentes de violences xénophobes, émeutes contre les autorités locales jugées corrompues, multiplication des mouvements de grève.

C'est largement pour répondre aux défis de ces inégalités spatiales qu'avait été mise en place la National Planning Commission qui a produit en 2011 un plan de développement (le National Development Plan, NDP, approuvé par l'ANC lors de son congrès de 2012). Le NDP proposait un traitement différencié des espaces, pensés en termes de corridors de développement rayonnant à partir du Gauteng et de «nœuds de compétitivité » autour des autres métropoles. Théoriquement cette politique territoriale devait être complétée par une action de redistribution au profit des anciens bantoustans. Critiqué par l'opposition de gauche, salué par les experts, ce programme a été de toutes manières mis en cause par la situation de crise politique récente, mais tout laisse penser que l'arrivée au pouvoir de Cyril Ramaphosa annonce un retour à la mise en œuvre du NDP, on en revient donc à l'hypothèse de Zapiro: «la fin d'une erreur ", donc la clôture d'une parenthèse...

Or il ne faut pas oublier que Jacob Zuma a été élu à la tête de l'État en 2009 et en 2014 en promettant de faire plus pour les exclus. Il était soutenu par la gauche de l'ANC, le parti communiste, les jeunesses de l'ANC et le mouvement syndical. Jacob Zuma a aussi perdu le soutien du parti communiste et des syndicats, et les événements de Marikana (cf. encadré 3), suivis par des grèves massives dans tous les secteurs industriels, ont été un déclencheur majeur de cette rupture politique. Ils ont aussi laissé un ANC divisé, et Cyril Ramaphosa n'a été élu à la tête du parti que d'extrême justesse contre l'ex-épouse de Jacob Zuma, Nkosazana Dlamini Zuma. La campagne interne à l'ANC a été d'une rare violence, et ce jusqu'à la base du parti : bien des branches locales n'ont même pas été en 
mesure de s'exprimer tant elles étaient fracturées entre les partisans des deux clans. Audelà, c'est la définition même du parti qui est aujourd'hui en jeu: est-il socialiste ou nationaliste noir? Est-il au service de la nouvelle élite et des classes moyennes émergentes ou des plus pauvres? Ces hésitations sont anciennes, elles remontent aux origines mêmes de l'ANC, mais le moment politique récent qui les a cristallisées date sans doute de 2012.

Encadré 3 - Le massacre de Marikana, août 2012

Cet événement dramatique a semblé signaler l'échec du compromis politique et social hérité de la négociation qui a conduit à l'abolition de l'apartheid. Le 16 août 2012, la police sud-africaine a abattu 34 mineurs grévistes de la mine de platine de Marikana (située dans la région de Rustenburg, au nord-ouest de Johannesburg) qui réclamaient une augmentation de salaire (78 autres grévistes ont été blessés par les tirs à balles réelles, la commission d'enquête a confirmé que beaucoup des balles meurtrières avaient été tirées dans le dos des victimes) avec l'appui d'un nouveau syndicat non-membre du COSATU. Cet événement majeur, la plus violente confrontation sociale depuis le début des années 1990, a évoqué douloureusement des souvenirs de répression policière des années de l'apartheid, et très précisément le «massacre de Sharpeville » du 21 mars 1960. La société Lonmin est un des acteurs économiques importants de l'exploitation du platine en Afrique du Sud, une des plus importantes activités minières du pays jusqu'à une récente chute des cours mondiaux. Les mineurs de la région, la platinum belt, un quart de siècle après la fin de l'apartheid, travaillent toujours dans le cadre d'un système hérité de la période coloniale puis de l'apartheid: le système du travail migrant. C'est-à-dire qu'il s'agit d'hommes migrants, sous contrat, en l'occurrence venant en majorité de l'ancien bantoustan du Transkei (aujourd'hui intégré dans la province du Cap de l'Est), secondairement de ressortissants du Lesotho et du Swaziland. Ils vivent soit dans des logements collectifs, les hostels, soit dans des bidonvilles proches des mines ou des baraques informelles des arrièrecours (backyard shacks) des maisons des townships voisins. Les événements de Marikana ont impliqué les principaux acteurs sociaux et politiques de l'Afrique du Sud post-apartheid y compris Cyril Ramaphosa, qui détient 9,1\% de Lonmin via le Shanduka Group, et dont il est avéré qu'il a influencé le gouvernement sur la conduite à tenir face à la grève de 2012. Ils ont mis en relief aux yeux de l'opposition et de nombreux citoyens la collusion entre le régime, les syndicats majoritaires et le patronat, jetant une ombre sur une démocratie longtemps jugée exemplaire : les conséquences politiques ont été dramatiques pour l'ANC et ses alliés.

19 Les questions qui se posent à l'ANC sont d'autant plus urgentes qu'il n'est plus, aujourd'hui, impossible d'envisager sa défaite lors des élections de 2019... L'alliance au pouvoir est contestée sur sa droite par la Democratic Alliance (DA), parti qui naguère recueillait essentiellement les votes des Blancs et des Coloreds (mais pèse aujourd'hui plus de $25 \%$ des voix à chaque élection) et sur sa gauche par les Economic Freedom Fighters (EFF, $8 \%$ des voix en 2016), parti de la gauche radicale fondé en 2013 qui appelle à la nationalisation des mines et des banques et à une redistribution massive des terres. Les élections municipales de 2016 ont démontré que l'ANC pouvait perdre. En effet, si l'ANC à l'échelle nationale a obtenu 53 \% des voix, la DA, soutenue par l'EFF, a pris le contrôle des grandes métropoles de Tswhane (Pretoria), Johannesburg, Nelson Mandela Bay (Port Elisabeth) et conservé la municipalité du Cap. campagnes contre l'ANC, dénonçant les compromissions des leaders du parti. La 
composition du nouveau gouvernement annoncé le 27 février par Cyril Ramaphosa prête le flanc à leurs critiques et montre la difficulté politique à laquelle est confrontée le nouveau chef de l'État : il doit tout à la fois donner des signes de rupture avec la période Zuma, et maintenir l'unité de l'ANC, tout en faisant revivre l'alliance avec le parti communiste (SACP) et le COSATU. Sur ce dernier point il semble avoir réussi, notamment en consultant ces partenaires sur la composition du gouvernement et en rappelant Blade Nzimande à un poste ministériel (ministre des transports). En revanche, si sept ministres trop impliqués dans des affaires de corruption ont bien été mis à l'écart, d'autres signes sont très ambigus. Des soutiens de Jacob Zuma restent dans le gouvernement ou $\mathrm{y}$ arrivent, c'est le cas de Malusi Gigaba qui de ministre des finances devient ministre de l'intérieur (Home affairs), ou de Nkosazana Dlamini-Zuma, candidate malheureuse à la présidence de l'ANC et ex-épouse de Jacob Zuma, qui obtient le portefeuille du "planning, monitoring and evaluation" auprès de la présidence. Ces personnalités vont devoir travailler avec les soutiens de Cyril Ramaphosa, comme Pravin Gordhan, qui revient au gouvernement au poste clé de ministre des entreprises publiques, Nhlanhla Nene à celui des finances (Nene et Gordhan avaient tous deux été évincés de ce même ministère pour leurs positions anti-corruption) ou Lindiwe Sisulu, qui obtient le poste de ministre des affaires étrangères.

Le point qui soulève politiquement le plus d'inquiétude est sans doute la nomination comme vice-président de David Mabusa (en décembre 2017 il avait été élu deputy president de l'ANC): pendant deux décennies il a été premier ministre de la province du Mpumalanga et à ce poste a été la cible de nombreuses accusations de corruption ; en revanche c'est bien grâce aux voix obtenues dans cette province que Ramaphosa a remporté les élections au sein de l'ANC, et beaucoup d'observateurs se sont inquiétés de l'étrange augmentation du nombre de votants dans le Mpumalanga lors du congrès du parti en décembre 2017...

\section{Alors, quel « post »?}

On comprend que bien au-delà du changement à la tête de l'État, événement finalement ponctuel et partiel, est ouvert un champ de réflexion sur l'Afrique du Sud d'aujourd'hui. Une nouvelle Afrique du Sud, par bien des aspects, mais aussi une Afrique du Sud qui restera héritée tant qu'aux échelles locale et nationale les inégalités sociales seront si fortement inscrites dans l'espace et s'auto-entretiendront dans un processus qui permet de parler, au sens propre, d'injustice spatiale. Si la période 1991-2008 a bien été un moment historique post-apartheid, marqué par la démocratisation sous l'égide de l'ANC, le début d'une émergence économique et les politiques de réconciliation nationale, comment qualifier la période actuelle ? Elle sera au moins post-Zuma, mais le blocage de l'émergence depuis la crise de 2008 et l'effritement du pouvoir de l'ANC semblent signaler un basculement dans un temps post-post-apartheid. Or Cyril Ramaphosa n'est-il pas le produit de la phase antérieure, celle qui a échoué à surmonter l'inertie des inégalités sociales et des structures spatiales héritées de l'apartheid? 


\section{BIBLIOGRAPHY}

Bond P., 2000. Elite Transition: From Apartheid to Neoliberalism in South Africa. Pietermaritzburg, University of KwaZulu-Natal Press, 318 p.

Bhorat H., Buthelezi M., Chipkin, I., Duma S., Mondi L., Peter C., Qobo M., Swilling M., Friedenstein H., 2017. Betrayal of the Promise : How South Africa is Being Stolen. Johannesburg, University of Johannesburg, Public Affairs and Research Institute - PARI, (State capacity research project), 66 p. https://pari.org.za/wp-content/uploads/2017/05/Betrayal-of-thePromise-25052017.pdf

Butler A., 2013. Cyril Ramaphosa. Johannesburg, Jacana Media, 500 p. (2nd revised edition)

Fine B., Rustomjee Z., 1996. The Political Economy of South Africa: From Minerals-Energy Complex to Industrialisation. Boulder (CO), Westview Press, 278 p.

Freund B., Witt H., (eds), 2010. Development Dilemmas in Post-Apartheid South Africa. Scottsville, University of KwaZulu-Natal Press, 424 p.

Gervais-Lambony P., 2017. Afrique du Sud. Les paradoxes de la nation arc-en-ciel. Paris, Le Cavalier Bleu éditions (Idées reçues), $174 \mathrm{p}$.

Jaglin S., Dubresson A., 2016. Eskom. Electricity and Technopolitics in South Africa. Cape Town, Juta/ UCT Press, $186 \mathrm{p}$.

Lodge T., 2014. Neo-Patrimonial Politics in the ANC. African Affairs, 113/450, p. 1-23.

Seekings J., Natrass N., 2011. State-Business relations and pro-poor growth in South Africa. Journal of International Development, 23, p. 338-357.

Southall R., 2010. South Africa 2010: From short-term success to long-term decline? In Daniel J., Naidoo P., Pillay D., Southall R., (eds), New South African Review 1 : 2010 : Development or Decline?, p. 1-21.

Tsheola J., Ledwaba M., Maphatane J., 2013. State-Owned Enterprises and State Capitalism: South Africa's Development Path Dilemma? In Delener N., Fuxman L., Rodrigues S., Rivera L. (eds), Globalizing Businesses for the next Century. Visualizing and Developping Contemporary Approaches to Harness Future Opportunities, Helsinki, GBTA, $15^{\text {th }}$ Annual Conference, July 2nd to 6th, Readings Book, 1392 p. (p. 1213-1223). http://gbata.org/wp-content/uploads/2013/06/ GBATA_2013_Readings_Book.pdf

Webster E., Adler G., 1999. Towards a class compromise in South Africa's 'double transition': bargained liberalization and the consolidation of democracy. Politics and Society (3), p. 347-385.

\section{AUTHORS}

\section{ALAIN DUBRESSON}

Alain Dubresson, adubress@parisnanterre.fr, est Professeur émérite et membre de l'UMR LAVUE. 


\section{PHILIPPE GERVAIS-LAMBONY}

Philippe Gervais-Lambony, philippe.gervais-lambony@parisnanterre.fr, est Professeur d'université à l'Université Paris-Nanterre et membre de l'UMR LAVUE. 